\title{
GLP-I mediates the modulating effect of thymoquinone on feeding behaviors in diabetic rats
}

This article was published in the following Dove Press journal:

Diabetes, Metabolic Syndrome and Obesity: Targets and Therapy

\author{
Shu Ping Lee' \\ Feng Yu Kuo ${ }^{1,2}$ \\ Juei-Tang Cheng ${ }^{3,4}$ \\ Ming Chang $\mathrm{Wu}^{\prime}$ \\ 'Department of Food Science, College of \\ Agriculture, National Pingtung University \\ of Science and Technology, Pingtung \\ 9120I, Taiwan; ${ }^{2}$ Cardiovascular Center, \\ Veterans General Hospital, Kaohsiung \\ City 8I362, Taiwan; ${ }^{3}$ Department of \\ Medical Research, Chi-Mei Medical \\ Center, Tainan City 71003, Taiwan; \\ ${ }^{4}$ Institute of Medical Science, College of \\ Health Science, Chang Jung Christian \\ University, Tainan City 7II0I, Taiwan
}

Background: Thymoquinone (TQ) is a safe nutrient isolated from the seeds or volatile oil extract of Nigella sativa. In addition to its benefits in glucose regulation, TQ improves feeding disorders in diabetic animals. Glucagon-like peptide-1 (GLP-1) analogs improve glycemic control and ameliorate obesity or hyperphagia. Therefore, the present study aimed to investigate the role of GLP-1 in TQ-induced anorexia.

Method: Type 2 diabetes was induced in rats by nicotinamide and streptozotocin injection. TQ was orally administered to diabetic rats at different doses for 45 days. Following TQ treatment, changes in serum glucose levels, GLP-1 concentration, body weight, food intake, and water intake were determined. To further explore the interaction between GLP-1 and TQ, the inhibitor of dipeptidyl peptidase 4, sitagliptin and the GLP-1 receptor antagonist exendin 9-39 (Ex 9-39) were separately administered to TQ- or vehicle-treated diabetic rats.

Results: TQ treatment attenuated hyperglycemia and reduced hyperphagy and water intake in streptozotocin-induced diabetic rats in a dose-dependent manner. Moreover, TQ treatment elevated plasma GLP-1 levels compared to those in control rats. The effects of TQ were enhanced by treatment with sitagliptin and reduced by the injection of Ex 9-39 into the brain. In contrast, similar treatment with another antioxidant (either ascorbic acid or $\mathrm{N}$-acetylcysteine) produced the same anorexic effect as TQ without changing the plasma GLP-1 levels in diabetic rats. Therefore, TQ attenuated hyperphagy while increasing plasma GLP-1 levels and had antioxidant-like effects.

Conclusion: TQ increased endogenous GLP-1 levels to reduce hyperphagy in diabetic rats. Keywords: thymoquinone, GLP-1, sitagliptin, body weight, food intake

\section{Introduction}

Thymoquinone (2-isopropyl-5-methyl-1,4-benzoquinone, TQ) is a widely used ingredient isolated from the seeds and volatile oil extract of black cumin (Nigella sativa). ${ }^{1}$ TQ is recognized as a safe nutrient, particularly when given orally to experimental animals. ${ }^{2}$ TQ elicits many effects, ${ }^{3}$ including immunomodulatory, anticancer, antidiabetic, antioxidant, anti-infertility, and anti-inflammatory activities and protects the liver, heart, and nervous system.

TQ exerts ameliorative and therapeutic effects on diabetic animal models, ${ }^{4,5}$ which could reduce hepatic glucose production. ${ }^{6}$ In the clinic, the hypoglycemic and hypolipidemic effects of black cumin in patients suffering from diabetes and metabolic syndrome have been reported. ${ }^{3}$ Additionally, TQ did not cause adverse effects on renal or hepatic function in diabetic patients. ${ }^{7}$ Therefore, TQ has been recommended as a food adjunct for diabetes. ${ }^{8}$ Interestingly, food intake was also reduced in diabetic animals following TQ administration. ${ }^{9}$ However, no report has
Correspondence: Juei-Tang Cheng City 71004, Taiwan

Tel +886 625 I 7864

Email jtcheng5503@gmail.com

Ming Chang Wu

Department of Food Science, College of Agriculture, National Pingtung University of Science and Technology, Neipu 9120I, Pingtung, Taiwan

Tel +88687703202

Email globalizationwu@yahoo.com.tw 
examined the mechanism(s) of the TQ-induced improvement of eating disorders in patients with diabetes.

Glucagon-like peptide-1 (GLP-1) is a gut hormone derived from the preproglucagon gene that is synthesized and released by intestinal L cells. ${ }^{10}$ GLP-1 and GLP-1 receptor expression was decreased with chronic hyperglycemia. ${ }^{11}$ Clinical study showed that GLP-1 exhibited a statistically significant decrease in type 2 diabetic compared with control subjects. $^{12}$ The intraperitoneal (IP) injection of GLP-1 decreased food intake in rodents. ${ }^{13,14}$ This finding is consistent with clinical reports that diabetic patients treated with GLP-1 or its stable receptor agonist progressively lose weight. ${ }^{15}$ Additionally, activation of the GLP-1 receptor (GLP-1R) in the central nervous system (CNS) was implicated in the regulation of food intake, ${ }^{16}$ mainly in the hypothalamic arcuate ${ }^{16}$ and paraventricular and supraoptic nuclei. ${ }^{17}$ The central administration of GLP-1-(7-36) amide inhibited food and water intake in rat. ${ }^{18}$

Changes in food and water intake due to GLP-1 modulation are similar to the effects of TQ. However, whether the effects of TQ on feeding behaviors in diabetic rats are mediated by GLP-1 is unknown. Therefore, the present study aimed to clarify these effects using type 2-like diabetic rats. First, we established a new model of type 2-like diabetes as described previously ${ }^{30}$ using the same doses of inducing agents except the change in fasting time. Then, three protocols were performed in the present study. The first experimental design aimed to confirm the effectiveness of TQ as a previous report $^{31}$ in the new model. Therefore, we used the same treatment period of 45 days. Otherwise, similar to a previous report $^{18}$, the results were effectively obtained in TQ-treated animals within 4 weeks, which was applied to the second experimental design. Finally, the role of the antioxidant-like effect was investigated in the third experimental design. Two antioxidants were used to treat for 45 days as that in the first experimental design. Changes in GLP-1 were then compared to clarify the role of antioxidant in the present study.

\section{Materials and methods}

TQ (purity >98\%) and exendin 9-39 (Ex 9-39) were obtained from Sigma Aldrich Chemical Co. (St. Louis, MO, USA). Sitagliptin phosphate (Merck, Cramlington, Northumberland, UK), which inhibits dipeptidyl peptidase4 (DPP-4) (Sigma Aldrich), was dissolved in normal saline.

\section{Animals}

Male Sprague-Dawley (SD) rats (a total of 120) weighing 250 to $280 \mathrm{~g}$ were obtained from the National Laboratory
Animal Center (Taipei, Taiwan). in the animals used for all experiments were maintained under anesthesia with $2 \%$ isoflurane to minimize suffering. The experimental protocols were approved by the Institutional Animal Ethics Committee $(105,051,901)$ of the Chi-Mei Medical Center. All experiments conformed to the Guide for the Care and Use of Laboratory Animals as well as the guidelines of the Animal Welfare Act. No mortality was observed in the experimental rats in the present study.

\section{The generation of diabetic rats}

To induce type 2-like diabetes, as described previously, ${ }^{19}$ rats were given an intravenous injection of streptozotocin (STZ, Sigma-Aldrich) at $65 \mathrm{mg} / \mathrm{kg}$ (dissolved in $5 \mathrm{mmol} / 1$ citrate buffer, $\mathrm{pH}$ 4.5) 15 mins after the IP injection of $230-\mathrm{mg} / \mathrm{kg}$ nicotinamide (NA, Sigma-Aldrich) dissolved in normal saline. Prior to induction, the rats were fasted for various times, as shown in the "Results" section. Then, the rats were given $5 \%$ dextrose instead of water on the first day of induction to avoid hypoglycemia. Fasting blood glucose levels were examined after 7 days. The rats were considered to be diabetic once they exhibited a plasma glucose level of no less than $200 \mathrm{mg} / \mathrm{dL}$, along with polyuria and other diabetic features.

\section{Experimental protocols Experimental design-I}

The rats were randomly divided into five groups as follows $(n=6)$ : group 1: normal control, group 2: diabetic control, group 3: diabetic rats receiving TQ $25 \mathrm{mg} / \mathrm{kg}$, group 4: diabetic rats receiving TQ $50 \mathrm{mg} / \mathrm{kg}$, and group 5: diabetic rats receiving TQ $100 \mathrm{mg} / \mathrm{kg}$. Rats in the normal control group and the diabetic control group were given an equal volume of normal saline. The administration of drugs or vehicle was carried out once daily for 45 days as described previously ${ }^{31}$ by oral gavage for the rats in all groups. The initial and final body weights of the rats in each group were recorded. Additionally, changes in daily food and water intake were measured at the end of the experiment.

\section{Experimental design-2}

In this section, the role of GLP-1 in mediating the effects of TQ was investigated. Rats were randomly assigned to 7 groups as follows $(n=6)$ : group I: normal control rats, group II: diabetic control rats (vehicle treated), group III: diabetic rats that received the oral administration of TQ $(50 \mathrm{mg} / \mathrm{kg} / \mathrm{day})$, group IV: diabetic rats that received the oral administration of TQ (50 mg/kg/day) and the ICV 
injection of Ex 9-39 (50 $\mu \mathrm{g}$ in $2 \mu \mathrm{L})$, group V: diabetic rats that received the oral administration of vehicle and the ICV injection of Ex 9-39 (50 $\mu \mathrm{g}$ in $2 \mu \mathrm{L})$, group VI: diabetic rats that received the oral administration of TQ $(50 \mathrm{mg} / \mathrm{kg} /$ day) and sitagliptin $(5 \mathrm{mg} / \mathrm{kg} /$ day $)$, and group VII: diabetic rats that received the oral administration of vehicle and sitagliptin (5 $\mathrm{mg} / \mathrm{kg} /$ day).

The TQ treatment course in each group lasted 4 weeks. ${ }^{20}$ The rats in group III received the ICV injection of Ex 9-39 to block GLP-1 receptors in the brain ${ }^{21} 30$ mins before TQ administration during the final week of treatment. The rats in group VI were orally treated with $5 \mathrm{mg} / \mathrm{kg} /$ day sitagliptin to inhibit the GLP-1 metabolic enzyme (DPP-4) ${ }^{22} 1 \mathrm{hr}$ before the oral administration of TQ daily for the last 2 weeks of treatment. The initial and final body weights of the rats in each group were recorded. Similarly, the daily food and water intake were measured before the end of the experiment.

\section{Intracerebroventricular (ICV) injection}

To understand the interaction between TQ consumption and GLP-1 receptor signaling in the CNS, the ICV infusion of Ex 9-39 was performed to block CNS GLP-1 activity. ${ }^{23}$ The rats were anesthetized with a mixture of isoflurane in oxygen (2\%) and placed in a Kopf stereotaxic instrument equipped with blunt ear bars. The stereotactic coordinates were $2.2 \mathrm{~mm}$ posterior to the bregma at a 7.5- $\mathrm{mm}$ depth from the dura mater and in the midline following the guide. ${ }^{24}$ The recovery period was at least 1 week; only rats that had regained more than $90 \%$ of their presurgery body weight were used in subsequent experiments.

The infusion cannula (22-gauge) attached to PE-10 tubing was inserted into the guide cannula and extended $0.5 \mathrm{~mm}$ beyond the guide. A $10.0 \mu \mathrm{L}$ Hamilton syringe was used to manually deliver saline or the tested agent over a 2-min period following our previous method. ${ }^{25}$ The infusion cannula was kept in place for an additional $1 \mathrm{~min}$ following infusion.

\section{Collection of blood and the estimation of biochemical parameters}

Blood samples $(200 \mu \mathrm{L})$ from fasting rats were collected from the tail vein under isoflurane anesthesia ${ }^{23}$ at the end of the feeding protocol. Then, the plasma was immediately separated by centrifuging at $13,000 \mathrm{rpm}$ for $10 \mathrm{mins}$ at $4^{\circ} \mathrm{C}$. All samples were stored at $-80^{\circ} \mathrm{C}$ until their analysis. The glucose levels were evaluated using a reagent from a glucose kit (Biosystems SA, Barcelona, Spain). Plasma insulin levels were determined using a rat insulin ELISA kit (Mercodia, Uppsala, Sweden). Insulin resistance was calculated using the homeostasis model assessment (HOMA-IR) method. HOMA-IR was then calculated as fasting glucose $(\mathrm{mmol} / \mathrm{L})$ $\times$ fasting insulin $(\mu \mathrm{U} / \mathrm{mL}) / 22.5{ }^{26}$ The GLP-1 levels were estimated using a commercial ELISA kit (Millipore).

\section{Measurement of body weight and food and water intake}

At the beginning and end of the study, the rats in each group were weighed using an electronic balance. Food intake and water intake were measured daily. Food intake was determined by measuring the difference between the preweighed food and the weight of the remaining food in the hopper and spilled food after $24 \mathrm{hrs}$. Water intake was measured by recording the quantity of water remaining in the feeding bottle.

\section{Administration of antioxidants to examine their effects on feeding behaviors in diabetic rats}

In an attempt to exclude the effects of antioxidants on the amelioration of diabetic like phenotypes, we administered two antioxidants, ascorbic acid (vitamin $\mathrm{C}^{27}$ and $\mathrm{N}$-acetylcysteine (NAC), ${ }^{28}$ to diabetic rats. The diabetic rats received ascorbic acid $(1 \mathrm{~g} / \mathrm{kg})^{29}$ or NAC $(0.5 \mathrm{~g} / \mathrm{kg})$ orally every day for 45 days, which was similar to the TQ treatment period. Then, feeding behaviors and plasma glucose or GLP-1 levels were determined as described above.

\section{Statistical analysis}

The results are indicated as the means \pm SDs of the indicated sample number (n) in each group. To investigate the effect of fasting time on glucose levels and insulin levels during induction of diabetes in rats and the changes in the body weight before and after TQ treatment with different dosage for 45 days, we conducted a two-way ANOVA, followed by Tukey's post hoc comparison. The rest of the results were compared using one-way ANOVA with Tukey's post-hoc test. The statistical analysis software SPSS 21 was used. A $P$-value of 0.05 indicated significance.

\section{Results}

\section{Effect of fasting time on the blood sugar response in diabetic rats}

In the present study, we compared the plasma glucose levels of diabetic rats that had fasted for various times. Hyperglycemia was more marked in diabetic rats with short 
A

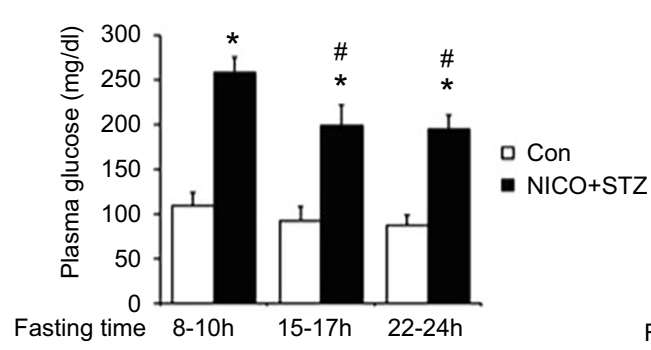

B



Figure I Effect of fasting time on glucose levels and insulin levels during the induction of diabetes in rats. After starvation for different time durations, the rats received an intraperitoneal injection of nicotinamide $(230 \mathrm{mg} / \mathrm{kg}$ ) 15 mins before the intravenous administration of STZ (65 mg/kg) (black column, Nicotinamide + STZ [NICO+STZ]). (A) Plasma glucose levels. (B) Plasma insulin levels. Values are expressed as means \pm SDs $(n=6)$. $* P<0.05$ compared with the control group (white column). $\# P<0.05$ compared with the vehicletreated diabetic group.

Abbreviations: STZ, streptozocin; Con, control.

fasting times than in diabetic rats with long fasting times (Figure 1A). Similarly, the insulin concentration was also significantly different after $8-10$ hrs of fasting (Figure 1B). Therefore, diabetic rats that had fasted approximately $8-10$ hrs were used as the model in the present study.

\section{Effects of TQ on feeding behaviors and body weights of diabetic rats}

The increased plasma glucose levels in diabetic rats were reversed by TQ treatment in a dose-dependent manner (Figure 2A). In the diabetic group, insulin sensitivity was markedly reduced, as revealed by increased HOMA-IR. TQ treatment decreased the HOMA-IR score compared with vehicle group (Figure 2B). The body weights at the beginning were similar in all groups. The body weights of diabetic rats in the control group were markedly reduced during the study period compared to those of rats in the normal control group. However, TQ treatment did not affect the body weights of diabetic rats (Figure 2C). Moreover, food and water intake were markedly increased in the diabetic rats compared to those in the normal control group. TQ decreased food (Figure 2D) and water intake (Figure 2E) in STZ-induced diabetic rats in a dose-dependent manner.

\section{Mediation of GLP-I in TQ-induced hypophagia in diabetic rats}

The ability of TQ to lower blood glucose levels was attenuated by the administration of Ex 9-39 in diabetic rats $(P<0.05$; Figure 3A). Additionally, the pretreatment of diabetic rats with Ex 9-39 reduced the TQ-induced increase in plasma GLP-1 levels $(P<0.05$; Figure 3B). The sitagliptin+TQ group showed a greater decrease in blood glucose and a marked increase in plasma GLP-1 levels compared to those in the TQ- or sitagliptin-treated groups (Figure 3A and B). In addition, TQ ameliorated polydipsia and polyphagia in diabetic rats; these effects were inhibited by Ex 9-39 treatment. In the presence of sitagliptin, ${ }^{22}$ the effects of TQ on food intake and water intake were increased (Figure 3C and D). In particular, the blood glucose level, GLP-1 concentration, food intake, and water intake of diabetic rats administered Ex 9-39 were no different than those of rats in the vehicle-treated group.

\section{Effects of antioxidants on feeding disorders in diabetic rats}

TQ exerts antioxidant-like effects. ${ }^{20}$ Similar to the effects of TQ, hyperglycemia was attenuated in diabetic rats that received NAC or ascorbic acid (Figure 4A). However, plasma GLP-1 levels were not modified by NAC or ascorbic acid (Figure 4B). Furthermore, changes in food intake (Figure 4C) and water intake (Figure 4D) were increased after the administration of these antioxidants in diabetic rats. Therefore, the role of endogenous GLP-1 in antioxidant-induced anorexic effects appears unlikely.

\section{Discussion}

TQ is the active principal ingredient in black cumin and has been recommended as a food adjunct for the management of diabetes. ${ }^{8}$ The present study found that TQ ameliorated feeding disorders in diabetic rats through endogenous GLP-1 activity.

Regarding the T2DM, many animal models have been developed and the model induced by nicotinamide with streptozotocin (NA-STZ) has widely been used. ${ }^{30-32}$ NASTZ rats are induced easily and quickly with a marked change in insulin, while hyperglycemia and hypoinsulinemia disorders meet the criteria for T2DM. ${ }^{33,34}$ But, the original NA-STZ model showed a hyperglycemia less than $198 \mathrm{mg} /$ 



B
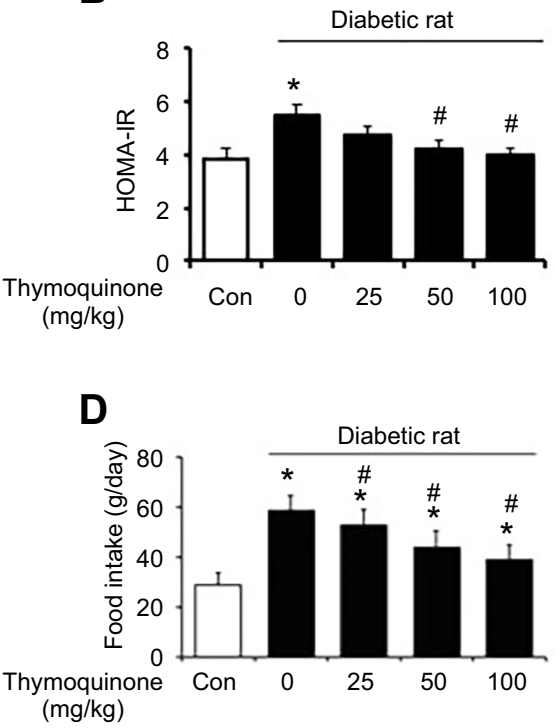

Figure 2 Chronic effects of thymoquinone (TQ) in normal and diabetic rats. (A) Plasma glucose levels in normal (white column) and diabetic (black column) rats. (B) Changes in HOMA-IR before (white column) and after (black column) treatment with different doses of TQ for 45 days. (C) Changes in body weight before (white column) and after (black column) treatment with different doses of TQ for 45 days. (D) Changes in food intake in normal (white column) and diabetic (black column) rats. (E) Changes in water intake in normal (white column) and diabetic (black column) rats. Values are expressed as means \pm SDs ( $n=6)$. *P<0.05 compared with the control group. $\# P<0.05$ compared with the vehicle-treated diabetic group.

Abbreviation: HOMA-IR, homeostatic model assessment of insulin resistance.

$\mathrm{dL}$ in rats. ${ }^{30}$ Therefore, researcher(s) changed the dose of NA or STZ during the induction of model for a higher plasma glucose in animals. ${ }^{35}$ In the present study, we demonstrated an alternative method without the change in NA or STZ dose as original report. ${ }^{30}$ To compare the effects of fasting period, we arranged for the rats to undergo fasting for various time periods during the induction of diabetes using the NA- or STZ-induced diabetic model, ${ }^{30,36}$ which was generated exactly as described in the original report. ${ }^{30}$ Fasting is usually used to minimize competition between STZ and glucose for low-affinity glucose transporter 2 on $\beta$ cells. ${ }^{37}$ The starvation period may also potentiate NA to protect $\beta$ cells against the cytotoxicity of STZ. ${ }^{35}$ Our results indicated that a short fasting time elevated the plasma glucose level in NA-STZ rats, reaching a level $(>260 \mathrm{mg} / \mathrm{dl})$ near that of the model induced by low doses of STZ alone. ${ }^{19}$ Therefore, we used this model in the present study. However, the degree of IR was not conducted, although the changes in HOMA-IR were significant. HOMA-IR is widely applied in clinical practice, but it remains questionable in animal research. ${ }^{19}$ Therefore, the degree of IR in this model should be investigated in the future.

Chronic treatment with TQ attenuated hyperglycemia and ameliorated feeding disorders in a dose-dependent manner in NA-STZ rats. It confirmed the results of a previous report ${ }^{38}$ involving the 45 -day oral intake. Additionally, it was also similar to a previous report. ${ }^{39,40}$ Rats in the diabetic group showed a significant increase in HOMA-IR, ${ }^{38}$ and treatment with TQ in NA-STZ diabetic rats decreased the HOMAIR. However, the decreased body weights observed in diabetic rats were not reversed by TQ. Diabetes is traditionally diagnosed by a severe loss in body weight, which appears primarily due to the unavailability of carbohydrates as an energy source ${ }^{41}$ or the resultant dehydration of the plasma fluid compartment. ${ }^{42}$ Recent 

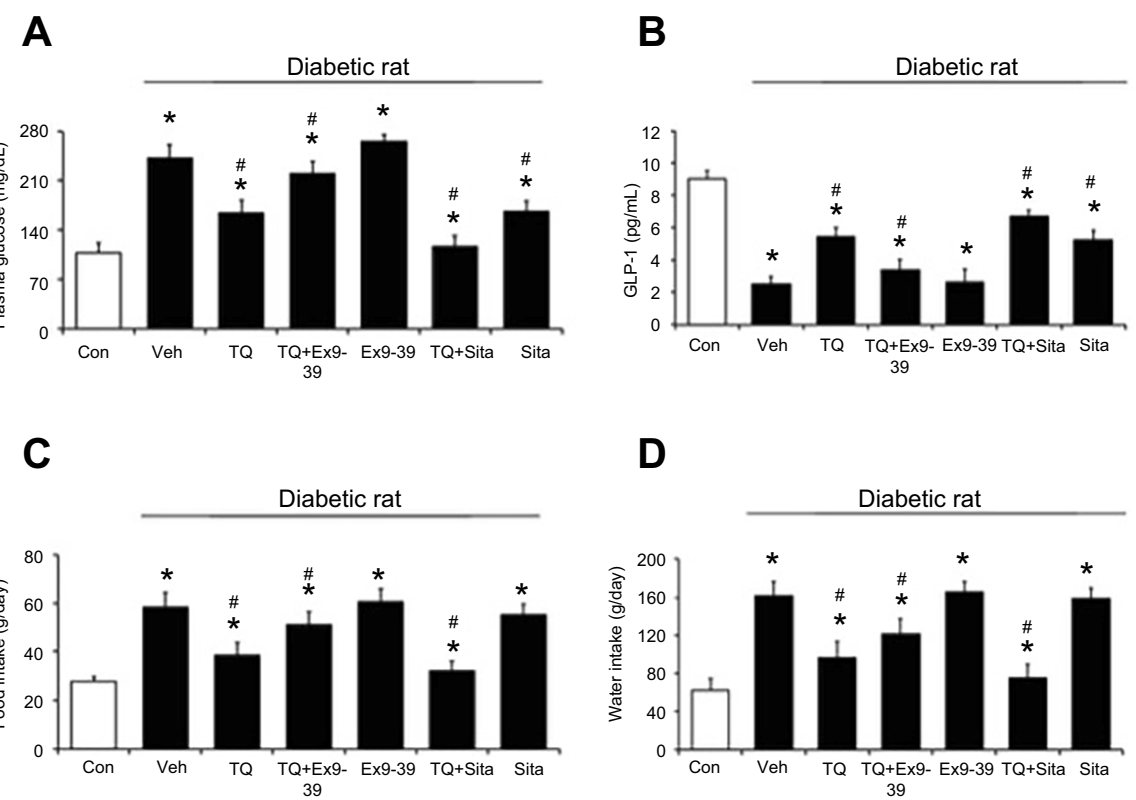

Figure 3 The chronic effects of thymoquinone (TQ) were modified by Ex 9-39 (GLP-I receptor antagonist) or sitagliptin (DPP-4 inhibitor) treatment. Diabetic rats were orally administered TQ $(50 \mathrm{mg} / \mathrm{kg} / \mathrm{day})$. (A) Plasma glucose levels. (B) Plasma GLP-I levels. (C) Food and (D) water intake in diabetic rats (black column). Values are expressed as the mean $\pm S D(n=6)$. $* P<0.05$ compared with the control group (white column). \#P<0.05 compared with the vehicle-treated diabetic group. Abbreviations: GLP-I, glucagon-like peptide-I; DPP-4, dipeptidyl peptidase-4.
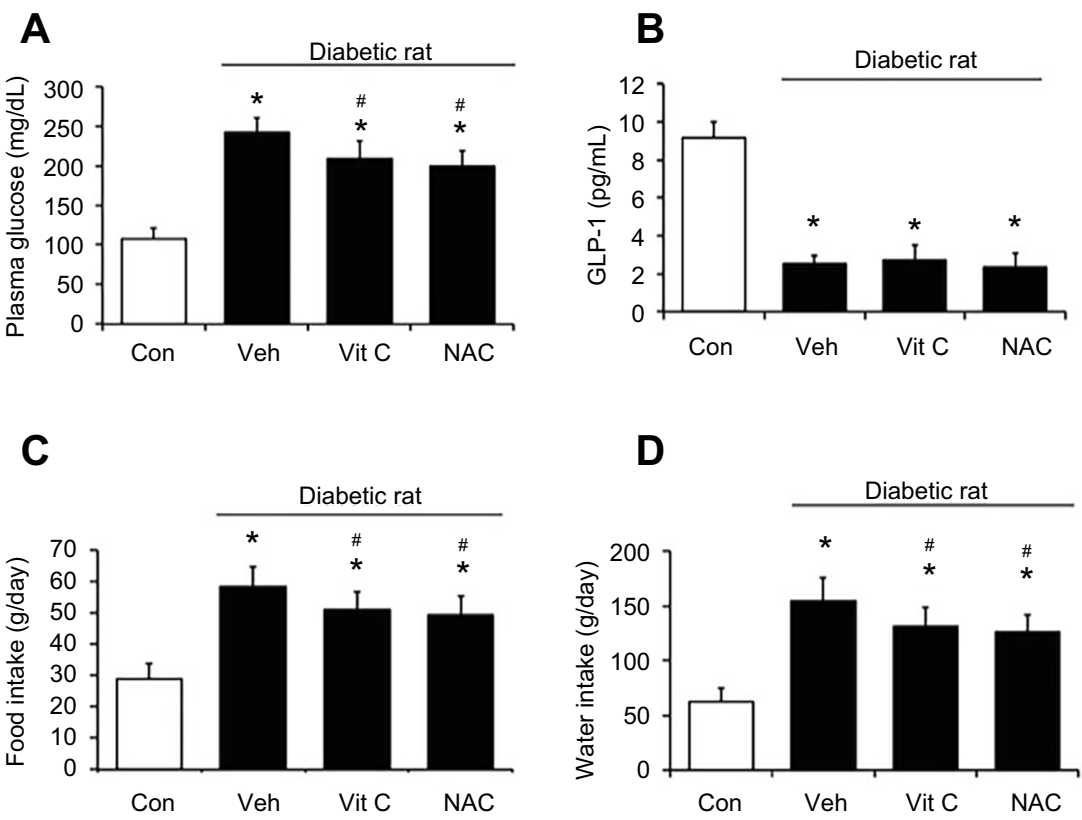

Figure 4 Effects of antioxidant treatment in rats. The diabetic rats (black column) received ascorbic acid (I g/kg) or NAC (0.5 g/kg) orally every day for 45 days. (A) Plasma glucose levels. (B) Plasma GLP-I levels. (C) Food and (D) water intake of the rats. Values are expressed as mean $\pm S D(n=6)$. $* P<0.05$ compared with the control group (white column). \#P<0.05 compared with the vehicle-treated diabetic group.

Abbreviation: NAC, N-acetylcysteine.

research has indicated that TQ improved islet revascularization and alleviated metabolic and hepatorenal disturbances in STZ-induced diabetic rats. ${ }^{43}$ In addition, TQ treatment also had a beneficial effect on glucose tolerance and insulin sensitivity in a diet-induced obesity mouse model of type 2 diabetes. $^{5}$
Ex 9-39 specifically blocks the GLP-1 receptor (GLP-1R), and it may inhibit the function of GLP-1 in the hypothalamus. ${ }^{44}$ The direct injection of Ex 9-39 into the brain changed eating behaviors in satiated rats. ${ }^{45}$ The injection of Ex 9-39 blocks the cerebral GLP-1R ${ }^{23}$ and was used to investigate the effects of TQ. After diabetic rats were 
administered Ex 9-39, the positive effects of TQ on both food intake and water intake were markedly reduced. Moreover, the effects of TQ on feeding disorders were enhanced in diabetic rats that received sitagliptin to inhibit DPP-4, a GLP-1-inactivating enzyme. ${ }^{46}$ The effective dose of sitagliptin administered was in accordance with previous reports. ${ }^{22,47}$ Additionally, the plasma GLP-1 level was markedly increased in diabetic rats that received sitagliptin compared to that in control rats. Therefore, the effects of TQ were mediated via endogenous GLP-1.

It was recently demonstrated that TQ exerts antioxidantlike effects. ${ }^{24}$ The antioxidant effect of TQ in cells might interact with the mitochondrial respiratory chain. ${ }^{48}$ TQ prevented diabetic induced cardiomyopathy via decrease of the cardiac malondialdehyde levels and significantly increased cardiac total superoxide dismutase. ${ }^{49}$ To determine the role of these potential antioxidant effects in the anorexic response to TQ, we compared the effects of two antioxidants in diabetic rats. Consistent with previous reports, the effects of the established antioxidants ascorbic $\operatorname{acid}^{27}$ and $\mathrm{NAC}^{28}$ were compared. Consistent with a previous report, ${ }^{43}$ the two antioxidants attenuated hyperglycemia and improved hyperphagia and water intake behaviors in diabetic rats. These effects were the same as those of TQ, with the exception that plasma GLP-1 levels were not influenced by NAC or ascorbic acid treatment. Antioxidants may ameliorate glucose homeostasis to improve disorders in diabetic rats. ${ }^{50}$ Therefore, the mediation of the anorexic effects of TQ treatment via endogenous GLP-1 is not entirely due to its antioxidant-like effects. Taken together, our results suggest that GLP-1 is released by TQ in the peripheral tissues and that it may reach the feeding center of the brain. The finding that the central administration of exogenous GLP-1 induced a marked reduction in food and water intake is consistent with previous findings in rats. ${ }^{18}$ Therefore, TQ likely ameliorates feeding disorders via endogenous GLP-1, at least in part.

\section{Conclusion}

In summary, we demonstrate that TQ treatment increased endogenous GLP-1 levels to ameliorate commonly observed feeding disorders in type 2-like diabetic rats. TQ is suitable for further development for the clinical treatment of obesity.

\section{Acknowledgments}

We thank Dr. Y Li and YC Chen for their helpful assistance in the preparation of the manuscript and collection of references and in the assistance of experiments, respectively. The present study was supported by a grant from the Chi-Mei Medical Foundation (CMFHT10503), Tainan City, Taiwan.

\section{Author contributions}

All authors contributed to data analysis, drafting or revising the article, gave final approval of the version to be published, and agreed to be accountable for all aspects of the work.

\section{Disclosure}

The authors report no conflicts of interest in this work.

\section{References}

1. Tiruppur Venkatachallam SK, Pattekhan H, Divakar S, Kadimi US. Chemical composition of Nigella sativa L. seed extracts obtained by supercritical carbon dioxide. J Food Sci Technol. 2010;47 (6):598-605. doi:10.1007/s13197-010-0109-y

2. Al-Attass SA, Zahran FM, Turkistany SA. Nigella sativa and its active constituent thymoquinone in oral health. Saudi Med J. 2016;37(3):235-244. doi:10.15537/smj.2016.3.13006

3. Tavakkoli A, Mahdian V, Razavi BM, Hosseinzadeh H. Review on clinical trials of black seed (Nigella sativa) and its active constituent, thymoquinone. J Pharmacopuncture. 2017;20(3):179-193. doi:10.3831/ KPI.2017.20.021

4. Sangi SM, Sulaiman MI, El-Wahab MF, Ahmedani EI, Ali SS. Antihyperglycemic effect of thymoquinone and oleuropein, on streptozotocin-induced diabetes mellitus in experimental animals. Pharmacogn Mag. 2015;11(Suppl 2):S251-257. doi:10.4103/09731296.166017

5. Karandrea S, Yin H, Liang X, Slitt AL, Heart EA. Thymoquinone ameliorates diabetic phenotype in diet-induced obesity mice via activation of SIRT-1-dependent pathways. PLoS One. 2017;12(9): e0185374. doi:10.1371/journal.pone.0185374

6. Abdelrazek HMA, Kilany OE, Muhammad MAA, Tag HM, Abdelazim AM. Black seed thymoquinone improved insulin secretion, hepatic glycogen storage, and oxidative stress in streptozotocin-induced diabetic male wistar rats. Oxid Med Cell Longev. 2018;2018:8104165. doi:10.1155/2018/8104165

7. Hayatdavoudi P, Khajavi Rad A, Rajaei Z, Hadjzadeh MA. Renal injury, nephrolithiasis and Nigella sativa: a mini review. Avicenna J Phytomed. 2016;6(1):1-8.

8. Mohammed A, Islam MS. Spice-derived bioactive ingredients: potential agents or food adjuvant in the management of diabetes mellitus. Front Pharmacol. 2018;9:893

9. Abduallah AM, Rashed AA, Gamaleldeen AK, Mohamed Sayed SR. The effect of nigella sativa extract (thymoquinone) on glucose insulin levels and body weight of induced diabetic female rats. Am J Life Sci. 2017;5(2):5.

10. Holst JJ. The physiology of glucagon-like peptide 1. Physiol Rev. 2007;87(4):1409-1439. doi:10.1152/physrev.00034.2006

11. Xu G, Kaneto H, Laybutt DR, et al. Downregulation of GLP-1 and GIP receptor expression by hyperglycemia: possible contribution to impaired incretin effects in diabetes. Diabetes. 2007;56 (6):1551-1558. doi:10.2337/db06-1033

12. Legakis IN, Tzioras C, Phenekos C. Decreased glucagon-like peptide 1 fasting levels in type 2 diabetes. Diabetes Care. 2003;26(1):252. doi:10.2337/diacare.26.1.252

13. Williams DL, Baskin DG, Schwartz MW. Leptin regulation of the anorexic response to glucagon-like peptide-1 receptor stimulation Diabetes. 2006;55(12):3387-3393. doi:10.2337/db06-0558 
14. Ronveaux CC, de Lartigue G, Raybould HE. Ability of GLP-1 to decrease food intake is dependent on nutritional status. Physiol Behav. 2014;135:222-229. doi:10.1016/j.physbeh.2014.06.015

15. Gutzwiller JP, Goke B, Drewe J, et al. Glucagon-like peptide-1: a potent regulator of food intake in humans. Gut. 1999;44(1):81-86. doi:10.1136/gut.44.1.81

16. NamKoong C, Kim MS, Jang BT, Lee YH, Cho YM, Choi HJ. Central administration of GLP-1 and GIP decreases feeding in mice. Biochem Biophys Res Commun. 2017;490(2):247-252. doi:10.1016/j.bbrc.2017.06.031

17. Tauchi M, Zhang R, D'Alessio DA, Stern JE, Herman JP. Distribution of glucagon-like peptide-1 immunoreactivity in the hypothalamic paraventricular and supraoptic nuclei. J Chem Neuroanat. 2008;36 (3-4):144-149. doi:10.1016/j.jchemneu.2008.07.009

18. Tang-Christensen M, Larsen PJ, Goke R, et al. Central administration of GLP-1-(7-36) amide inhibits food and water intake in rats. Am J Physiol. 1996;271(4 Pt 2):R848-856. doi:10.1152/ajpregu.1996.271.4.R848

19. Chao PC, Li Y, Chang CH, Shieh JP, Cheng JT, Cheng KC. Investigation of insulin resistance in the popularly used four rat models of type-2 diabetes. Biomed Pharmacother. 2018;101:155-161. doi:10.1016/j.biopha.2018.02.084

20. Demiroren K, Basunlu MT, Erten R, Cokluk E. A comparison of the effects of thymoquinone, silymarin and $\mathrm{N}$-acetylcysteine in an experimental hepatotoxicity. Biomed Pharmacother. 2018;106:1705-1712. doi:10.1016/j.biopha.2018.07.125

21. Williams DL, Baskin DG, Schwartz MW. Evidence that intestinal glucagon-like peptide-1 plays a physiological role in satiety. Endocrinology. 2009;150(4):1680-1687. doi:10.1210/en.2008-1045

22. Badole SL, Chaudhari SM, Bagul PP, et al. Effect of concomitant administration of L-glutamine and cycloart-23-ene-3beta, 25-diol (B2) with sitagliptin in GLP-1 (7-36) amide secretion, biochemical and oxidative stress in streptozotocin - nicotinamide induced diabetic Sprague Dawley rats. PLoS One. 2013;8(8):e72817. doi:10.1371/ journal.pone.0072817

23. Kanoski SE, Fortin SM, Arnold M, Grill HJ, Hayes MR. Peripheral and central GLP-1 receptor populations mediate the anorectic effects of peripherally administered GLP-1 receptor agonists, liraglutide and exendin-4. Endocrinology. 2011;152(8):3103-3112. doi:10.1210/ en.2011-0174

24. Jessen L, Smith EP, Ulrich-Lai Y, et al. Central nervous system GLP-1 receptors regulate islet hormone secretion and glucose homeostasis in male rats. Endocrinology. 2017;158(7):2124-2133. doi:10.1210/en.2016-1826

25. Chang TK, Niu CS, Cheng JT. Activation of alpha 1-adrenoceptors to lower cerebrocortical neuropeptide Y (NPY)-like immunoreactivity in rats receiving pargyline treatment. Neurosci Lett. 1996;218(3):161-164.

26. Kuo SC, Chung HH, Huang CH, Cheng JT. Decrease of hyperglycemia by syringaldehyde in diabetic rats. Horm Metab Res. 2014;46 (1):8-13. doi:10.1055/s-0033-1351274

27. Padayatty SJ, Katz A, Wang Y, et al. Vitamin C as an antioxidant: evaluation of its role in disease prevention. $J$ Am Coll Nutr. 2003;22 (1):18-35.

28. Fiordaliso F, Bianchi R, Staszewsky L, et al. Antioxidant treatment attenuates hyperglycemia-induced cardiomyocyte death in rats. $J \mathrm{Mol}$ Cell Cardiol. 2004;37(5):959-968. doi:10.1016/j.yjmcc.2004.07.008

29. Young IS, Torney JJ, Trimble ER. The effect of ascorbate supplementation on oxidative stress in the streptozotocin diabetic rat. Free Radic Biol Med. 1992;13(1):41-46.

30. Masiello P, Broca C, Gross R, et al. Experimental NIDDM: development of a new model in adult rats administered streptozotocin and nicotinamide. Diabetes. 1998;47(2):224-229.

31. Matsumoto T, Wakabayashi K, Kobayashi T, Kamata K. Alterations in vascular endothelial function in the aorta and mesenteric artery in type II diabetic rats. Can J Physiol Pharmacol. 2004;82(3):175-182. doi:10.1139/y04-002
32. Rabbani SI, Devi K, Khanam S. Role of pioglitazone with metformin or glimepiride on oxidative stress-induced nuclear damage and reproductive toxicity in diabetic rats. Malays J Med Sci. 2010;17(1):3-11.

33. Kumar M, Sharma S, Vasudeva N. In vivo assessment of antihyperglycemic and antioxidant activity from oil of seeds of brassica nigra in streptozotocin induced diabetic rats. Adv Pharm Bull. 2013;3 (2):359-365. doi:10.5681/apb.2013.058

34. Saravanan R, Pari L. Succinic acid monoethyl ester prevents oxidative stress in streptozotocin-nicotinamide-induced type2 diabetic rats. J Basic Clin Physiol Pharmacol. 2006;17(2):115-132.

35. Szkudelski T. Streptozotocin-nicotinamide-induced diabetes in the rat. Characteristics of the experimental model. Exp Biol Med (Maywood). 2012;237(5):481-490. doi:10.1258/ebm.2012.011372

36. Pari L, Sankaranarayanan C. Beneficial effects of thymoquinone on hepatic key enzymes in streptozotocin-nicotinamide induced diabetic rats. Life Sci. 2009;85(23-26):830-834. doi:10.1016/j.lfs.2009.10.021

37. Chaudhry ZZ, Morris DL, Moss DR, et al. Streptozotocin is equally diabetogenic whether administered to fed or fasted mice. Lab Anim. 2013;47(4):257-265. doi:10.1177/0023677213489548

38. Morakinyo AO, Samuel TA, Adekunbi DA. Magnesium upregulates insulin receptor and glucose transporter-4 in streptozotocin-nicotinamide-induced type-2 diabetic rats. Endocr Regul. 2018;52(1):6-16. doi:10.2478/enr-2018-0002

39. Prabhakar P, Reeta KH, Maulik SK, Dinda AK, Gupta YK. Protective effect of thymoquinone against high-fructose diet-induced metabolic syndrome in rats. Eur J Nutr. 2015;54(7):1117-1127. doi:10.1007/ s00394-014-0788-7

40. El-Mahmoudy A, Shimizu Y, Shiina T, Matsuyama H, El-Sayed M, Takewaki T. Successful abrogation by thymoquinone against induction of diabetes mellitus with streptozotocin via nitric oxide inhibitory mechanism. Int Immunopharmacol. 2005;5(1):195-207. doi:10.1016/j.intimp.2004.09.001

41. Al-Shamaony L, Al-Khazraji SM, Twaij HA. Hypoglycaemic effect of Artemisia herba alba. II. Effect of a valuable extract on some blood parameters in diabetic animals. J Ethnopharmacol. 1994;43(3):167-171.

42. AI-Saaidi JAA, Kareem HMA, AI-Tameemi WTM. Antihyperglycemic effects of thmoquinone in diabetic rats. Bas $J$ Vet Res. 2014;13(2):180-192.

43. El-Shemi AG, Kensara OA, Alsaegh A, Mukhtar MH. Pharmacotherapy with thymoquinone improved pancreatic beta-cell integrity and functional activity, enhanced islets revascularization, and alleviated metabolic and hepato-renal disturbances in streptozotocin-induced diabetes in rats. Pharmacology. 2018;101(1-2):9-21. doi: $10.1159 / 000480018$

44. Kinzig KP, D'Alessio DA, Seeley RJ. The diverse roles of specific GLP-1 receptors in the control of food intake and the response to visceral illness. $J$ Neurosci. 2002;22(23):10470-10476.

45. Meeran K, O'Shea D, Edwards CM, et al. Repeated intracerebroventricular administration of glucagon-like peptide-1-(7-36) amide or exendin-(9-39) alters body weight in the rat. Endocrinology. 1999;140(1):244-250. doi:10.1210/endo.140.1.6421

46. Herman GA, Bergman A, Stevens C, et al. Effect of single oral doses of sitagliptin, a dipeptidyl peptidase- 4 inhibitor, on incretin and plasma glucose levels after an oral glucose tolerance test in patients with type 2 diabetes. $J$ Clin Endocrinol Metab. 2006;91 (11):4612-4619. doi:10.1210/jc.2006-1009

47. Badole SL, Bagul PP, Mahamuni SP, et al. Oral L-glutamine increases active GLP-1 (7-36) amide secretion and improves glycemic control in stretpozotocin-nicotinamide induced diabetic rats. Chem Biol Interact. 2013;203(2):530-541. doi:10.1016/j.cbi.2013.02.006

48. Martinovich GG, Martinovich IV, Vcherashniaya AV, Cherenkevich SN. Thymoquinone, a biologically active component of Nigella sativa, induces mitochondrial production of reactive oxygen species and programmed death of tumor cells. Biophysics. 2016;16:963. doi:10.1134/S0006350916060154 
49. Atta MS, El-Far AH, Farrag FA, Abdel-Daim MM, Al Jaouni SK, Mousa SA. Thymoquinone attenuates cardiomyopathy in streptozotocin-treated diabetic rats. Oxid Med Cell Longev. 2018;2018:7845681. doi:10.1155/2018/7845681
50. Chang YC, Chuang LM. The role of oxidative stress in the pathogenesis of type 2 diabetes: from molecular mechanism to clinical implication. Am J Transl Res. 2010;2(3):316-331.

\section{Publish your work in this journal}

Diabetes, Metabolic Syndrome and Obesity: Targets and Therapy is an international, peer-reviewed open-access journal committed to the rapid publication of the latest laboratory and clinical findings in the fields of diabetes, metabolic syndrome and obesity research. Original research, review, case reports, hypothesis formation, expert opinion and commentaries are all considered for publication. The manuscript management system is completely online and includes a very quick and fair peer-review system, which is all easy to use. Visit http://www.dovepress.com/testimonials.php to read real quotes from published authors. 